\title{
Influence of minimal subgroups on the product of smooth groups
}

\author{
A. M. Elkholy *, M. H. Abd El-Latif \\ Mathematics Department, Faculty of Science, Beni Suef University, Beni-Suef 62511, Egypt \\ *Corresponding author E-mail: aelkholy9@yahoo.com
}

Copyright (C2015 A. M. Elkholy and M. H. Abd El-Latif. This is an open access article distributed under the Creative Commons Attribution License, which permits unrestricted use, distribution, and reproduction in any medium, provided the original work is properly cited.

\begin{abstract}
A maximal chain in a finite lattice $L$ is called smooth if any two intervals of the same length are isomorphic. We say that a finite group $G$ is totally smooth if all maximal chains in its subgroup lattice $L(G)$ are smooth. In this article, we study the product of finite groups which have a permutable subgroup of prime order under the assumption that the maximal subgroups are totally smooth.
\end{abstract}

Keywords: Permutable subgroups; Smooth groups; Subgroup lattices.

\section{Introduction}

Only finite groups wiil be considered in this paper. Notation is standard and is taken mainly from Doerk and Hawkes [2]. In addition, for a fixed group $G$, the maximal length of the subgroup lattice $L(G)$ will be denoted by $n$, and $\pi(G)$ will denote the set of all distinct primes dividing $|G|$.

A subgroup $H$ of a group $G$ is permutable in $G$ if it permutes with every subgroup of $G$. A subgroup $H$ of a group $G$ is said to be permutable in a subgroup $K$ of $G$ if it permutes with every subgroup of $K$. This concept was introduced by Asaad and Shaalan [3]. A group $G$ is called smooth if $G$ has a maximal chain of subgroups in which any two intervals of the same length are isomorphic. Finite smooth groups have been studied by Schmidt $[4,5]$. A group $G$ is said to be totally smooth if every maximal chain of subgroups is smooth. Finite totally smooth groups have been studied in [1].

A lattice $L$ is said to be complemented if every element of $L$ has a complement in $L$. Recall that a $P$-group is either an elementary abelian group of order $p^{n}$ for a prime $p$, or a semidirect product of an elementary abelian normal subgroup $P$ of order $p^{n-1}$ for a prime $p$ and a cyclic $q$-group inducing a power automorphism group on $P$, where $p$ and $q$ are different primes (see $[6 ;$ p. 49]).

The purpose of this article is studying the product of finite totally smooth groups which have a permutable subgroup of prime order under the assumption that the maximal subgroups are totally smooth. Clearly, the structure of groups with $n \leq 2$ is well known. So we assume that $n \geq 3$.

\section{Main results}

The following Lemma will be used in the sequel: 
Lemma 2.1 A group $G$ is totally smooth if and only if one of the following holds:

(i) $G$ is cyclic of prime power order.

(ii) $G$ is a P-group.

(iii) $G$ is cyclic of square free order (See [1]; Theorem 1).

In this article, we will deal only with groups whose order is divided at least by two primes. So we assume firstly that $|\pi(G)|=2$.

Theorem 2.2 Let $G=H K$ be the product of its proper subgroups $H$ and $K$ with $n \geq 3$ and $|\pi(G)|=2$. Assume that all maximal subgroups of $G$ are totally smooth. Let $N$ be a minimal normal subgroup of $H$. If $N$ is permutable in $K$, then one of the following holds:

(i) $G$ is a nonabelian P-group.

(ii) $n=3$ and $|G|=p^{2} q$, where $p$ and $q$ are distinct primes in $\pi(G)$.

(iii) $G=P Q$, where $P$ is a cyclic Sylow p-subgroup of order $p^{2}$ and $Q$ is an elementary abelian normal subgroup of $G$ of $\operatorname{order} q^{e}(e>1)$.

Proof. Since all maximal subgroups of $G$ are totally smooth, it follows by Lemma 2.1, that $H$ and $K$ are cyclic of prime power orders, P-groups, or cyclic of square free order. Let $P$ be a Sylow p-subgroup of $G$ and $Q$ be a Sylow $q$-subgroup of $G$. We have the following cases:

case 1 . $H$ is cyclic. It follows by Lemma 2.1 that either $|H|=p^{\alpha}$ with $\alpha \geq 1$ or $|H|=p q$ with $p \neq q$.

Assume that $|H|=p^{\alpha}$ with $\alpha \geq 1$. Hence $|N|=p$.

Suppose, further, that $K$ is cyclic of prime power order. Since $|\pi(G)|=2,|K|=q \beta$ with $q \neq p$. It follows that $H$ is complemented in $G$. Let $K_{1}$ be a proper subgroup of $K$. If $|H|=p$, then $H=N$ is permutable in $K$ and $H K_{1}$ is a proper subgroup of $G$. By hypothesis, $H K_{1}$ is totally smooth and hence Lemma 2.1 shows that $H K_{1}$ is a nonabelian $P$-group or cyclic of square free order. Since $H$ and $K$ are cyclic, it follows that $\left|H K_{1}\right|=p q$. Hence $K$ would be of order $q^{2}$ as $K_{1}$ is any proper subgroup of $K$. Then $|G|=p q^{2}$ and (ii) holds. So assume that $|H|>p$. Hence $H$ has a permutable subgroup $N$ in $K$. By hypothesis, $N K$ would be a proper subgroup of $G$ and by using Lemma 2.1, it is cyclic of order $p q$ or a nonabelian P-group. Since $K$ is cyclic, $|K|=q$.

Let $p$ be the largest prime in $\pi(G)$. Since $K$ is cyclic, we get $H G$. If $H_{1}$ is a maximal subgroup of $H$, it follows that $H_{1} G$ and hence $H_{1} K<G$. Since $H_{1}$ is cyclic, it follows by hypothesis and Lemma 2.1 that $H_{1} K$ would be of order $p q$. Then $|G|=p^{2} q$ and we are done. So let $q$ be the largest prime in $\pi(G)$. Then $K G$ and hence $|G|=p^{2} q$. Now suppose that $K$ is cyclic of order $p q$, Hence $|Q|=q$. If $n=3,|G|=p^{2} q$ and we are done. So let $n \geq 4$. It follows that $|H| \geq p^{2}$. As $H$ is cyclic and $P$ is totally smooth, $P$ would be cyclic. If $p>q$, $P G$ which implies that every subgroup of $P$ is normal in $G$. Then there exists a proper subgroup $L$ of $G$ with $|L|=p^{2} q$ which is not totally smooth, a contradiction. Thus $p<q$. Since $P$ is cyclic, $Q G$. Once again, $|G|=p^{2} q$ and $n=3$, a contradiction.

Final, suppose that $K$ is a $P$-group. It follows that $K$ is elementary abelian or a nonabelian P-group. Assume that $K$ is elementary abelian of order $q^{\beta}$ with $\beta>1$.

If $|H|=p, H$ is permutable in $K$ and hence $H K_{1}$ is a maximal subgroup of $G$ where $K_{1}$ is a maximal subgroup of $K$. Since $H K_{1}$ is totally smooth, we have by Lemma 2.1, that $H K_{1}$ is cyclic of order pq or a nonabelian P-group. If $\left|H K_{1}\right|=p q$, then $n=3$ and $G$ would be of order $p q^{2}$. Otherwise, $H K_{1}$ is a nonabelian P-group with $p<q$. Hence $K G$. Since $K_{1}$ is any maximal subgroup of $K$ and $H K_{1}$ is a nonabelian P-group, $H$ does not centralize any subgroup of $K$. Then $G$ is a nonabelian P-group of order $p q^{\beta}(\beta>1)$ and (i) holds. So assume that $|H|>p$. Hence $H$ has a permutable subgroup $N$ in $K$. By Lemma 2.1, NK would be cyclic of order pq or a nonabelian P-group. If $N K$ would be of order $p q,|K|=p$ which contradicts that $\beta>1$. Thus $N K$ would be a nonabelian $P$-group with $p<q$. Since $H$ is cyclic, $K G$. If $H_{1}$ is a maximal subgroup of $H, H_{1} K$ is totally smooth proper subgroup of $G$. Since $\beta>1, H_{1} K$ would be a nonabelian $P$-group which implies that $\left|H_{1}\right|=p$ and hence $|H|=p^{2}$. If $K$ has a normal subgroup in $G, H$ would be of order $p$, we get a contradiction since $|H|>p$. Thus $K$ is a minimal normal subgroup of $G$ and (iii) holds. Now assume that $K$ is a nonabelian P-group.

If $n=3,|G|=p^{2} q$ and we are done. So assume that $n \geq 4$. Obviously, $Q<K$. If $p>q, P G$. We get by Lemma 2.1, $P$ is cyclic or elementary abelian. If $P$ is cyclic, $n=3$ which contradicts that $n \geq 4$. Thus $P$ is elementary abelian. Then $H$ would be of order $p$. Since $K$ is a nonabelian P-group, there exists a proper subgroup $L$ of $K$ with $|L|=p$ and $L K$. As $P$ is elementary abelian, $L P$ and hence $L G$. Since $n \geq 4$, we get $p^{2}|||| G / L \mid$. As $G / L$ is totally smooth, $G / L$ would be a nonabelian $P$-group. Then $Q$ does not centralize any subgroup of $P$ and every subgroup of $P$ is normal in $G$. Therefore, $Q$ induces a nontrivial power automorphism on $P$ and hence $G$ is a nonabelian P-group. So let $p<q$.

Assume that $|H|>p$. Then $P$ would be cyclic and hence $Q G$. Clearly, $L_{1} Q<G$ where $L_{1}$ is a maximal subgroup of $P$. Since $q>p,|L|=p$ and hence $|P|=p^{2}$. If $Q$ has a proper subgroup $Q_{1}$ such that $Q_{1} G$, we get $Q_{1} P<G$. By hypothesis, $Q_{1} P$ is totally smooth. By applying Lemma 2.1, $|P|=p$, a contradiction. Thus $Q$ is a minimal 
normal subgroup of $G$ and (iii) holds. So assume that $|H|=p$. It follows that $H Q<G$ as $G=H K$ is a product of its proper subgroups $H$ and $K$. If $Q$ has a proper subgroup $Q_{1}$, we get $Q_{1} H Q$. Since $Q_{1} K$, it follows that $Q_{1} G$. Clearly, $Q_{1} H$ is a totally smooth proper subgroup of $G$. By Lemma 2.1, $H$ would be of order $p$, a contradiction. Thus $Q$ would be of order $q$ and hence $n=3$ which contradicts that $n \geq 4$.

Thus $H$ is cyclic of order pq. Then every minimal subgroup of $H$ is permutable in $K$. It is clear that if $n=3$, we are done. So assume that $n \geq 4$.

Suppose that $K$ is of prime power order and let $N<H$ with $(|N|,|K|)=1$. So we can assume that $|K|=p^{\beta}$ and hence $|N|=q$. Clearly, $N K \leq G$. Since $n \geq 4,|K| \geq p^{2}$. If $G=N K$, we get a proper subgroup $U$ of $G$ containing $H$ with $p^{2}|| U \mid$ which is not smooth since $H$ is cyclic of order $p q$, a contradiction. Thus $N K<G$. By hypothesis and Lemma 2.1, $N K$ would be nonabelian P-group of order $p^{e} q(e \geq 2)$. Hence $P$ would be elementary abelian and $|Q|=q$. Similar, there exists a non-totally smooth subgroup $V$ of $G$ containing $H$ with $p^{2}|| V \mid$ which contradicts our hypothesis. Thus $K$ is a nonabelian $P$-group of order $p^{\alpha} q$ or cyclic of order $p q$.

Suppose that $K$ is cyclic of order pq. Since $G=H K$ is a product of its proper subgroups $H$ and $K$, it follows that $n=3$ which contradicts that $n \geq 4$. Thus $K$ is a nonabelian P-group of order $p^{\alpha} q(p>q)$. Since $G=H K$, we get a normal subgroup $N$ of $H$ with $N K$. By hypothesis, $N K \leq G$. If $N K=G$ and since $H$ is cyclic, we get $n=3$ which contradicts our assumption that $n \geq 4$. Thus $N K<G$. If $q^{2}|| N K \mid$, then $[N K / 1]$ is not smooth since $K$ is a nonabelian P-group which contradicts our hypothesis. Thus $N$ would be of order $p$. It follows that $N$ would be normal in $N K$ as $K$ is a nonabelian P-group. Once again, there exists a subgroup of $G$ containing $H$ which is not smooth, a contradiction.

case 2. $H$ is a P-group.

It follows that $H$ is elementary abelian or a nonabelian P-group. Suppose, first, that $H$ is elementary abelian of order $p^{\alpha}$ with $\alpha>1$. Then $H$ has a permutable subgroup $N$ in $K$. It is clear that if $n=3$, then $|G|=p^{2} q$ and we are done. So let $n \geq 4$.

Assume that $K$ is cyclic of order $q^{\beta}, \beta \geq 1$. Since $n \geq 4$, we get by hypothesis that $N K$ is a totally smooth proper subgroup of $G$. It follows by lemma 2.1 that $N K$ is cyclic of order pq or a nonabelian P-group. If $N K$ is cyclic, $N N K$ and $|K|=q$. Hence $N G$. Since $n \geq 4$, we get $|H|>p^{2}$. By hypothesis and lemma 2.1, $G / N$ would be a nonabelian $P$-group with $p>q$. Since $|H|>p^{2}$, it follows that $H$ has a permutable subgroup $N_{1}$ in $K$ with $N_{1} G$. Then there is a subgroup of $G$ containing $N N_{1}$ and $K$ which is not smooth, a contradiction. Thus $N K$ is a nonabelian P-group for any minimal normal subgroup $N$ of $H,|K|=q$, and every subgroup of $H$ is normal in $G$. Since $K$ does not centralize any subgroup of $H$, it follows that $G$ is a nonabelian P-group and we are done.

Let $K$ be cyclic of order $p q$. Since $|H|>p$ and $n \geq 4$, there exists a permutable subgroup $N$ of $H$ with $N K$. It follows that $[N K / 1]$ is not smooth, a contradiction. Thus $K$ is a P-group. If $K$ is elementary abelian group of order $q^{\beta}$ with $\beta>1$. Since $H$ has a permutable subgroup $N$ in $K$, it follows that $N K$ is a proper subgroup of $G$. Our hypothesis and lemma 2.1 show that $N K$ would be a nonabelian P-group with $q>p$ as $\beta>1$. Since $N$ is any minimal normal subgroup of $H$, there exists a subgroup $Q_{1}$ of $Q$ of order $q$ which is normal in $G$. Then $H Q_{1}$ is a proper subgroup of $G$. Since $|H|>p$, it follows by lemma 2.1 that $[H Q 1 / 1]$ is not smooth; a contradiction as $p<q$. Thus $K$ is a nonabelian P-group.

Suppose first that $|K|=p^{\beta} q,(p>q)$. It follows that $p$ is the largest prime dividing $|G|$ and hence $Q$ would be of order $q$. Then $P G$ and $P$ is elementary abelian. Then $G$ has a normal subgroup $P_{1}$ of order $p$ with $P_{1}<K$. By hypothesis and lemma 2.1, $G / P_{1}$ would be a nonabelian $P$-group. Since $P_{1}$ is any minimal subgroup of $P, G$ would be a nonabelian P-group.

Now consider $|K|=q^{\beta} p$. Hence $Q<K$ and $p$ is the smallest prime dividing $|G|$. Since $|H|>p, H$ has a permutable subgroup $N$ of $H$. Among all such minimal normal subgroups $N$ of $H$, choose $N$ such that $N K$. Hence $N Q$ is a proper subgroup of $G$ which is totally smooth. Applying lemma 2.1, NQ is cyclic of order pq or a nonabelian $P$-group $(q>p)$. Then $Q N Q$ for each $N<H$ as $H$ is elementary abelian. Hence $Q G$.

If $|Q|=q$ and since $n \geq 4$, then $G$ has a proper subgroup $U$ containing $Q$ such thatp ${ }^{2}|| U \mid$ which is not totally smooth. Since $p<q$, we get a contradiction. Thus $|Q|>q$ and hence $N Q$ is a nonabelian P-group. Let $Q_{1}$ be a maximal subgroup of $Q$. Clearly, $Q_{1} N Q$ for each $N<H$ and so $Q_{1} G$. Similar, we get a contradiction since $p^{2}|| G / Q_{1} \mid$ and $\left[G / Q_{1}\right]$ is not totally smooth. Thus assume that $H$ is a nonabelian P-group of order $p^{\alpha} q$ with $\alpha \geq 1$. It follows that $H$ has a normal subgroup $N$ of order $p$.

If $K$ would be cyclic of order $q^{\beta}$, we get $n=3$ and $|G|=p q^{2}$ since $G=H K$. Thus assume that $K$ is cyclic of order $p^{\beta}$. Clearly, $|Q|=q$ and so $P G$. If $P$ is cyclic, $|G|=p^{2} q$ and we are done. So suppose that $n \geq 4$ and $P$ is elementary abelian. Hence $|K|=p$. Since $G / N$ is totally smooth, $G / N$ would be nonabelian P-group and hence $Q$ does not centralize any subgroup of $P$. Therefore, $G$ is a nonabelian P-group. So assume that $K$ is cyclic of order pq. Since $G=H K$ is the product of its proper subgroups $H$ and $K$, there exists a minimal normal subgroup $N$ of $H$ such that $N K$. Hence $N K<G$ and by lemma 2.1 we get $[N K / 1]$ is not totally smooth, a contradiction. Thus 
$n=3$ and (ii) holds.

ocmFinal, consider $K$ is a P-group. Hence $K$ is elementary abelian or a nonabelian P-group of order $p^{\beta} q$.

Assume that $K$ is elementary abelian of order $q^{\beta}$ with $\beta>1$. As $H$ is a nonabelian P-group, $H$ has a normal subgroup $N$ of order $p$ and by hypothesis $N K$ is a subgroup of $G$. Since $G=H K$ is a product of its proper subgroup $H$ and $K, N K$ would be a proper subgroup of $G$ which is totally smooth. Since $p>q$, it follows by lemma 2.1 that $|K|=q$ which contradicts our assumption that $\beta>1$. Thus $K$ is elementary abelian of order $p^{\beta}$. Similar, we get $|Q|=q$ and $P$ is elementary abelian normal Sylow p-subgroup of $G$. Therefore, $G / N$ is a nonabelian $P$-group and $Q$ does not centralize any p-subgroup of $P$. Hence $Q$ induces a nontrivial power automorphism on $P$. Then $G$ is a nonabelian $P$-group and we are done. To complete the proof, $K$ would be a nonabelian $P$-group of order $p^{\beta} q$. Hence $N N K$ and so NG. Similar, G/N is a nonabelian P-group and consequently $Q$ would be of order $q$. Once again, $G$ would be a nonabelian P-group. This completes our proof.

Now we are in a position to prove the case when $|\pi(G)| \geq 3$.

Theorem 2.3 Let $G=H K$ be the product of its proper subgroups $H$ and $K$ with $n \geq 3$ and $|\pi(G)| \geq 3$. Assume that all maximal subgroups of $G$ are totally smooth. If every minimal normal subgroup of $H$ is permutable in $K$, then one of the following holds:

(i) $G$ is cyclic of square free order.

(ii) $n=3$ and $|G|=p q r$, where $p, q$, and $r$ are distinct primes in $\pi(G)$.

\section{Proof.}

As all maximal subgroups of $G$ are totally smooth, we get by Lemma 2.1, that $H$ and $K$ are cyclic of prime power orders, P-groups, or cyclic of square free order. Let $N$ be a minimal normal subgroup of $H$. We have the following cases:

case 1. $H$ is cyclic.

It follows that either $|H|=p^{\alpha}$ with $\alpha \geq 1$ or $H$ is of order $p_{1} p_{2} \ldots p_{m}$ where $p_{i} \neq p_{j}(i \neq j)$ and $i, j=1,2, \ldots m$. Let $|H|=p^{\alpha}$ with $\alpha \geq 1$. Since $|\pi(G)| \geq 3,|\pi(K)| \geq 2$. So we can assume that either $K$ is cyclic of square free order or a nonabelian P-group of order $q^{\beta} r,(q>r)$.

Suppose first that $K$ is a nonabelian $P$-group of order $q^{\beta} r,(q>r)$ and let $K=Q R$ where $Q$ is a Sylow $q$-subgroup of $K$ and $R$ is a Sylow r-subgroup of $K$. If $|H|>p$, we get by hypothesis that $N K<G$ where $N$ is a normal subgroup of $H$ of order $p$. Furthermore, $|\pi(N K)|=3$. Since $K$ is a nonabelian P-group, we have by lemma 2.1 that $[N K / 1]$ is not totally smooth which contradicts our hypothesis. Thus $H$ would be of order $p$. Since all maximal subgroups of $G$ are supersolvable, it follows that $G$ is solvable and so $G$ has a Sylow basis. Hence $H Q<G$. If $|Q|=q$, then $|G|=p q r$ and (ii) holds. So let $|Q|>q$. Hence by lemma 2.1, HQ would be a nonabelian P-group $(q>p)$. Then there exists a proper subgroup $Q_{1}$ of $Q$ which is normal in $H Q$. Since $K$ is a nonabelian $P$-group, $Q_{1} K$. Therefore, $Q_{1} G$. Clearly, $H R<G$. Then $Q_{1} H R<G$. Since $R$ does not centralize $Q_{1}$ and $\left|\pi\left(Q_{1} H R\right)\right|=3$, we get $\left[Q_{1} H R / 1\right]$ is not totally smooth which contradicts our hypothesis. Thus $K$ is cyclic of square free order. Let $P_{i}$ be a Sylow $p_{i}$-subgroup of $G$ with $p \neq p_{i}$. By the solvability of $G$ and since $|H|=p^{\alpha}$, HP $P_{i}$ is a subgroup of $G$. By hypothesis and lemma 2.1, $H$ would be of order $p$ as $H$ is cyclic. Hence the Sylow subgroups of $G$ are of prime orders. If $G$ is abelian, then $G$ is cyclic. Otherwise, $|\pi(G)|=3$ and $G$ would be of order pqr.

Now assume that $H$ is a cyclic of order $p_{1} p_{2} \ldots p_{m}$ with $(m \geq 2)$. Then there exists a minimal normal subgroup $N$ of $H$ with $N K$ such that $N K \leq G$. Since $|\pi(G)| \geq 3, K$ would be a P-group or cyclic of square free order or cyclic of prime power order.

Suppose first that $K$ is cyclic of order $p^{\alpha}$. It follows that $N K$ is a totally smooth proper subgroup of $G$. By lemma 2.1, NK would be cyclic of square free order or a nonabelian P-group. As $K$ is cyclic, $|K|=p$. If $n=3$, then $G$ is cyclic or (ii) holds. Thus assume that $n \geq 4$. Since $G$ is solvable, $P P_{j}<G$ where $P$ is a Sylow $p$-subgroup of $G$ and $P_{j}$ is a Sylow $p_{j}$-subgroup of $G\left(p \neq p_{j}\right)$. We argue that $|P|=p$.

Suppose for a contradiction that $|P|>p$. We have by lemma 2.1 that $P P_{j}$ is a nonabelian $P-g r o u p\left(p>p_{j}\right)$. Then there exists a proper subgroup $L$ of $P$ which is normal in $P P_{j}$ and hence it is normal in $G$ as $P_{j}$ is any $S y l o w$ $p_{j}$-subgroup of $G\left(p \neq p_{j}\right)$. Since $|\pi(G)| \geq 3$, it follows that $L P_{i} P_{j}<G$ where $p_{j} \neq p_{i} \neq p$. We get by hypothesis and lemma 2.1 that $L P_{i} P_{j}$ is cyclic, a contradiction since $P_{j}$ does not centralize L. Thus $|K|=|P|=p$ and so the Sylow subgroups of $G$ are of prime orders. As $n \geq 4,|\pi(G)| \geq 4$. Hence $P P_{1} P_{2}$ is a proper subgroup of $G$ and by lemma 2.1, it would be cyclic. Thus $P_{i} G, i=1,2$. By applying lemma 2.1, $G / P_{i}$ would be cyclic as $\left|\pi\left(G / P_{i}\right)\right| \geq 3$. Then $G \leq P_{1} \cap P_{2}=1$ hence $G$ is abelian. Therefore $G$ is cyclic of square free order.

Now Let $K$ be a cyclic of square free order such that $|\pi(K)| \geq 2$. Since $G=H K$ is a product of its proper subgroups $H$ and $K$, it follows that there exists a minimal subgroup $N$ of $H$ with $N K$. Hence $N K \leq G$. Obviously, if $p^{2}|| G \mid$ for some prime $p \in \pi(G)$ and since $G$ is solvable, we get a normal subgroup L of order $p$. Similar, L $P_{1} P_{j}$ is a cyclic 
subgroup of $G$. Then by lemma 2.1, $\left[P P_{j} / 1\right]$ is not smooth, a contradiction. Thus the Sylow subgroups of $G$ are of prime orders.

Assume first that $G=N K$ and let $K_{1}$ be a maximal subgroup of $K$. Hence $N K_{1}<G$. By hypothesis and lemma 2.1, $N K_{1}$ is a nonabelian P-group or cyclic. If $|\pi(G)|=3$, we are done since $N K_{1}$ is of square free order. So let $|\pi(G)| \geq 4$. It follows that $N K_{1}$ would be cyclic and hence every Sylow subgroup of $K$ centralizes $N$ as $K_{1}$ is any maximal subgroup of $K$. Then $G$ is abelian and hence it is cyclic of square free order. Now assume that $N K<G$. Since the Sylow subgroups of $G$ are of prime orders and $|\pi(K)| \geq 2, N K$ would be cyclic of square free order and $|\pi(G)| \geq 4$. Once again, $G$ is cyclic.

Consider $K$ is a P-group. Hence it is elementary abelian or a nonabelian P-group. Suppose first that $K$ is a nonabelian $P$-group of order $p^{\alpha} q, p>q$. If $N K<G$, we have a contradiction since $K$ is a nonabelian $P$-group and $|\pi(N K)|=3$. Thus $N K=G$ and hence $|\pi(G)|=3$.

Suppose, for a contradiction, that $n \geq 4$. Since $N$ is of prime order and $(|N|,|K|)=1$, it follows that $|G|$ would be divided by $p^{2}$ where $p$ is the largest prime in $\pi(K)$. Let $K_{1}$ be a maximal nonabelian $P$-subgroup of $K$. It follows that $N K_{1}$ is a proper subgroup of $G$. As $\left|\pi\left(N K_{1}\right)\right|=3$, we have by lemma 2.1 that $N K_{1}$ would be cyclic. Since $K_{1}$ is a nonabelian P-group, we get a contradiction. Thus $n=3$ and we are done. So assume that $K$ is elementary abelian of order $p^{\beta}, \beta>1$. It follows $N K$ is a totally smooth proper subgroup of $G$ where $N$ is a minimal subgroup of $H$. Since $|K|>p$, we get by lemma 2.1 that $N K$ would be a nonabelian $P$-group where $p$ is the largest prime in $\pi(N K)$. Let $L<K$. Then $L N K$ and hence $L G$ as $N$ is any minimal subgroup of $H$. Let $N_{1}$ and $N_{2}$ be minimal subgroups of $H$ such that $N_{1} \neq N_{2}$. Clearly, $L N_{1} N_{2}<G$. Since $\left|\pi\left(L N_{1} N_{2}\right)\right|=3, L N_{1} N_{2}$ would be cyclic which contradicts that $N_{i}$ does not centralize $L(i=1,2)$. Thus $|K|=p$, a contradiction as $\beta>1$.

case 2. $H$ is a P-group. Hence $H$ is elementary abelian or a nonabelian P-group.

Suppose, first, that $H$ is elementary abelian of order $p^{\alpha}$ with $\alpha>1$. Therefore $H$ has a permutable subgroup $N$ in $K$. Hence $K$ is a nonabelian $P$-group or cyclic of square free order. Suppose that $K$ is a nonabelian P-group. We get $|\pi(G)|=3$ and $(|N|,|K|)=1$. Then $N K$ would be cyclic which contradicts our choice of $K$. Thus $K$ is cyclic of square free order. Let $P$ be a Sylow p-subgroup of $G$. As $P$ is totally smooth and $H$ is elementary abelian p-subgroup, it follows that $P$ would be elementary abelian. If $p$ would be dividing $|K|$, we get $G$ has a normal subgroup $N$ of order $p$. Hence $|\pi(G / N)| \geq 3$ which implies that $G / N$ would be cyclic of square free order since $G / N$ is totally smooth. Then $|P|=p^{2}$. Let $Q$ be a Sylow $q$-subgroup of $G$ with $q \neq p$. Since $G$ is solvable, $P Q$ is a subgroup of $G$. Since $Q$ centralizes $N,[P Q / 1]$ is not smooth which contradicts our hypothesis. Thus $p|K|$. It follows that $N K<G$. Similar, we get a contradiction as $|H|>p$. Thus $H$ is a nonabelian P-group of order pap $p_{1}$, $p>p_{1}$. Hence $|N|=p$.

Consider, first, that $K$ is a nonabelian $P$-group. Then $|\pi(G)| \leq 4$. Let $P_{i}$ be a Sylow $p_{i}$-subgroups of $G$ with $p_{i} \neq p$. Since $G$ is solvable, we have $P P_{i}$ is a totally smooth proper subgroup of $G$ which is a nonabelian $P$-group or cyclic. If $|P|>p, P P_{i}$ would be a nonabelian $P$-group and $N P P_{i}$. Then $N G$ as $P_{i}$ is any Sylow $p_{i}$-subgroups of $G$. Hence $N P_{1} P_{2}<G$, for $i=1,2$. Since $P_{i}$ does not centralize $N$, it follows by lemma 2.1 that $\left[N P_{1} P_{2} / 1\right]$ is not smooth which contradicts our hypothesis. Thus $|P|=p$.

If $|\pi(G)|=4$, we get by hypothesis and lemma 2.1 that $P K$ is a cyclic subgroup of $G$ and hence PPK. Since PH, we get $P G$. Once again $\left[P P_{1} P_{2} / 1\right]$ is not smooth, a contradiction. Therefore $|\pi(G)|=3$ and $p$ would be the largest prime in $\pi(G)$ which implies that $n=3$ and (ii) holds.

Assume that $K$ is elementary abelian of order $p_{2}^{\beta}$. Once again, if $n=3$, we get $|G|=p p_{1} p_{2}$ and we are done. So let $n \geq 4$. By hypothesis, $N K$ is a totally smooth subgroup of $G$. Suppose that $p$ is the largest prime in $\pi(G)$. Then $N N K$ and $|K|=p_{2}$. Hence $N G$. As $G / N$ is totally smooth, it follows by lemma 2.1 that $G / N$ is a nonabelian $P$-group or cyclic of square free order. If $G / N$ is a nonabelian P-group, $|\pi(G)|=3$ which implies that $n=3$, a contradiction. Thus $G / N$ is cyclic with $|\pi(G / N)| \geq 3$ as $n \geq 4$. Since $P_{1}$ does not centralize $N$, $[N P 1 K / 1]$ is not smooth which contradicts our hypothesis. Therefore $p_{2}$ is the largest prime in $\pi(G)$ which implies that $|P|=p$ since $P_{2}$ is a totally smooth subgroup of $G$. Suppose, for a contradiction, that $|K|>p_{2}$. Since $G$ is solvable, we have that $G$ has a Sylow basis and since $|K|>p_{2}$, it follows that $K$ has a normal subgroup $L$ in $G$. Hence $L H<G$. Since $H$ is a nonabelian P-group, $[L H / 1]$ is not smooth which contradicts our hypothesis. Therefore $|K|=p_{2}$ and hence $n=3$, a contradiction as $n \geq 4$.

To complete the proof, assume that $K$ is cyclic. Suppose first that $K$ is a cyclic of square free order and let $P_{j}$ be Sylow $p_{j}$-subgroups of $G$ with $p_{j} \neq p(j=1,2, \ldots, m)$. The solvability of $G$ shows that, $P P_{j}<G$. We argue that $|P|=p$. If $|P|>p$, then $P P_{j}$ is a nonabelian $P$-group and $\left|P_{j}\right|=p_{j}$ for each $j=1,2, \ldots, m$. Then $N P P_{j}$ and hence $N G$. It follows that $N P_{1} P_{2}<G$. Then by lemma 2.1, it is cyclic; a contradiction since $P_{1}$ does not centralize $N$. Thus $|P|=p$. Suppose, for a contradiction, that $n \geq 4$. Since $H$ is a nonabelian $P$-group, $G$ has a proper subgroup $M$ containing $H$ with $|\pi(M)|=3$ which is not smooth. Thus $n=3,|G|=p p_{1} p_{2}$ and we are done. Now assume $K$ is cyclic of prime power order. Then $(|N|,|K|)=1$. Since $N K$ is a totally smooth proper subgroup of $G, K$ 
would be of prime order. If $K G$, we get $|P|=p$ where $P$ is a Sylow $p$-subgroup of $G$. Then $n=3$ and (ii) holds. Otherwise, $p$ is the largest prime in $\pi(G)$. Once again if $N<P$, we get a contradiction. Thus $|P|=p$ and $n=3$. This completes our proof.

\section{Conclusion}

In this paper, we proved the following result:

Theorem 3.1 Let $G=H K$ be the product of its proper subgroups $H$ and $K$ with $n \geq 3$ and $|\pi(G)| \geq 2$. Assume that all maximal subgroups of $G$ are totally smooth. If every minimal normal subgroup of $H$ is permutable in $K$, then one of the following holds:

(i) $G$ is a nonabelian P-group.

(ii) $G$ is cyclic of square free order.

(iii) $n=3$ and $|G|=p q r$, where $p$ and $q$ are not necessary distinct primes in $\pi(G)$.

(iv) $G=P Q$, where $P$ is a cyclic Sylow p-subgroup of order $p^{2}$ and $Q$ is an elementary abelian normal subgroup of $G$ of order $q^{e}(e>1)$.

Clearly, the proof of Theorem 3.1 is included in both Theorem 2.2 and Theorem 2.3.

\section{References}

[1] A. M. Elkholy, "On totally smooth groups", Int. J. Algebra, Vol.1, No.2, (2007), pp.63-70.

[2] K. Doerk, T. Hawkes, Finite soluble groups. Walter de Gruyter, Berlin-New York, (1992).

[3] M. Asaad, Shaalan, "On the supersolvability of finite groups", Arch. Math, 53, (1989), pp.318-326.

[4] R. Schmidt, "Smooth groups", Geometriae Dedicata, 84, (2001), pp.183-206.

[5] R. Schmidt, "Smooth p-groups", J. Algebra, 234, (2000), pp.533-5390.

[6] R. Schmidt, Subgroup lattices of groups. Walter de Gruyter, Berlin-New York, Berlin-New York, (1994). 